\title{
Estudios métricos de la bibliografía básica de los planes de enseñanza del curso de Biblioteconomía de la Universidad Estadual Paulista
}

Metric studies of the basic bibliography used at the LIS course plans of education from the University of São Paulo State

\section{Ely Francina Tannuri de Oliveira (1), João Batista Ernesto de Moraes (2) y Fábio Orsi Meschini (3) \\ Departamento de Ciência da Informação, Faculdade de Filosofia e Ciências, Universidade Estadual Paulista, Brasil (1) etannuri@flash.tv.br, (2) Correo electrónico: jota@marilia.unesp.br}

(3) fabioorsi@marilia.unesp.br

\begin{abstract}
Resumen
Este estudio tiene por objetivo analizar y evaluar la bibliografía básica de los planes de enseñanza del curso de licenciatura en Biblioteconomía de la Universidad Estadual Paulista, Unesp - Campus de Marília. Así, se pretende diagnosticar si hay una naturaleza más humanística o más técnica del curso, contribuyendo en la búsqueda de la identidad y del auto conocimiento del curso. A través del análisis de citas se puede evaluar el desempeño y el impacto de diversos tipos de documentos (libros, revistas, actas de congreso, etc.). Esta investigación consiste en un estudio métrico que utilizó la técnica de análisis de la bibliografía indicada, a través del análisis de los siguientes puntos: tipología de los documentos, lenguas referenciadas, autores citados, vida media de la literatura, formato de los documentos, procedencia de los autores, universidades más citadas, autores más citados, y periódicos más citados. Los resultados parciales revelaron que los libros y capítulos de libro presentaron mayor porcentaje de frecuencia de citas, así como también el formato de documento impreso, aunque el formato electrónico estuviera también siendo contemplado en todas las asignaturas, pero con menor frecuencia. El análisis de procedencia de autoría, identificó la mayoría de autores como brasileños, como la lengua más citada el portugués y la vida media de la literatura inferior a 8 años.
\end{abstract}

Palabras-clave: Estudios métricos. Análisis de la bibliografía. Planes de Enseñanza.

\section{Introducción}

Las referencias bibliográficas, como el propio nombre dice, dan el "norte" del curso, representan la filosofía y las bases epistemológicas de este, y tienen como objetivos orientar tanto la formación general como la formación específica. Así, una referencia citada en lengua extranjera

\begin{abstract}
This study has as objective analyzing and evaluating the basic bibliography used in the Bibliotheconomy course schedule from the University of São Paulo State, Unesp- Marília Campus. Thus, it is explored if the course has a more humanistic or technical nature, contributing to its search of identity and selfknowledge. Thanks to citation analysis it is possible to evaluate the performance and the impact of diverse types of documents (books, magazines, papers, etc.). The of following items are analyzed: typology of documents, languages, cited authors, average life of literature, format of documents, origin of the authors, universities, authors and newspapers more cited. The partial results revealed that books and chapters represent the greatest percentile of citation frequency, as well printed documents, although electronic ones are also being contemplated in all the disciplines, however with lesser frequency. The authorship analysis identified a majority as Brazilian authors; Portuguese as the more cited language; and 8 years as the average life of literature.
\end{abstract}

Keywords: Metric studies. Bibliographic analysis. Educational plans. 
su nivel de actualización, como también de la inserción de la literatura en al ámbito nacional e internacional.

Con la intención de contribuir a la reflexión sobre la licenciatura, este estudio tiene por objetivo analizar y evaluar la bibliografía básica de los planes de enseñanza del curso de licenciatura en Biblioteconomía de la Universidad Estadual Paulista, Unesp - Campus de Marília. El objetivo específico fue delinear el perfil de la bibliografía citada en los planes de enseñanza de los docentes de las asignaturas de formación específica, o sea, aquellas dirigidas a la formación profesional del alumno, partiendo del presupuesto que el estudio de este perfil ofrece bases para la comprensión de las bases filosóficas, epistemológicas y educacionales que sustentan el curso.

Así, se pretende diagnosticar la naturaleza del curso, contribuyendo a la búsqueda de la identidad y del autoconocimiento, insertándose así en una postura auto-evaluativa, dinámica y de constante transformación.

Varias áreas de conocimiento humano han realizado estudios sobre la bibliografía básica o mínima de sus cursos. Sin embargo, pocos son los estudios realizados sobre esta temática en el área de Biblioteconomía. En los estudios métricos, se destaca la parte de análisis de la bibliografía citada, toda vez que esta técnica auxilia en la cuantificación y análisis de la información registrada, al analizar los estándares y frecuencias de citas hechas por los diferentes docentes, así como la relación entre los documentos citados. Lo que justifica el análisis de las referencias citadas es la consideración de que una producción científica no es una entidad solitaria, sino que está inmersa en la literatura sobre el tema. A través del análisis de citas se puede evaluar el desempeño y el impacto de diversos tipos de documentos (libros, revistas, actas de congreso, etc.), así como de las autorías y corrientes de pensamiento en uso.

El análisis de citas es una parte de los estudios métricos que investiga las relaciones entre los documentos que citan y los que son citados: autoría, título, origen geográfico, año, lengua de publicación, etc

\section{Procedimientos metodológicos}

Se usó un abordaje cuantitativo, en el cual se buscó explorar el análisis y la interpretación de los resultados obtenidos. Los objetos de estudio fueron las referencias bibliográficas de veintitrés planes de enseñanza de las asignaturas de formación específica del curso de Bibliotecono- mía de la Universidad Estadual Paulista UNESP, Marília, constantes en el currículo en el primer semestre del año de 2007. Las unidades de análisis fueron esas referencias, considerándose los siguientes aspectos: tipología de los documentos, lenguas referenciadas, autores citados, vida media de la literatura, formato de los documentos, procedencia de los autores, autores más citados y revistas más citadas.

\section{Resultados y Discusión}

Como resultado de la recolección, se obtuvieron trescientas nueve referencias en un total de 23 asignaturas de formación específica del currículo, impartidas en el curso de Biblioteconomía en el primer semestre de 2007. El número medio de referencias por asignatura fue de 13,43, siendo que la asignatura Catalogación Automatizada fue la que presentó mayor número de referencias, con 89 de un total de 309 referencias, completando el $28,80 \%$ del total, seguida de Catalogación con 59 referencias, representando el $19,09 \%$ y luego enseguida Introducción a la Ciencia de la Información, con 20 referencias, representando el $6,47 \%$ del total.

\begin{tabular}{lrr}
\hline Asignaturas & $N^{\circ}$ & $\%$ \\
\hline Catalogación Automatizada & 89 & 28,80 \\
Catalogación & 59 & 19,09 \\
Introducción a la Ciencia de la Información & 20 & 6,47 \\
Introducción a la Editoración & 16 & 5,18 \\
Estudio de Usuarios & 13 & 4,21 \\
Indización & 11 & 3,56 \\
Marketing en Unidades de Información & 09 & 2,91 \\
Diseminación de la Información & 09 & 2,91 \\
Organización y métodos en & 08 & 2,59 \\
Biblioteconomía & 08 & 2,59 \\
Análisis Documental & 07 & 2,26 \\
Condensación Documental & & \\
Sistema de Gerenciamiento Automático & 07 & 2,26 \\
de Unidades de Información & 07 & 2,26 \\
Formación y Actuación Profesional & 07 & 2,26 \\
Evolución de los Soportes de Información & 06 & 1,94 \\
Fuentes de Información & 06 & 1,94 \\
Lenguajes Documentales Jerárquicas & 05 & 1,62 \\
Práctica Profesional en Biblioteconomía & 04 & 1,62 \\
Lingüística y Documentación & 04 & 1,29 \\
Lenguajes Documentales Alfabéticas & 04 & 1,29 \\
Lectura Documental & 03 & 0,97 \\
Planeamiento en Unidades de & 02 & 0,65 \\
Información & & \\
Desarrollo de Colecciones & \multicolumn{2}{|c}{} \\
Normalización Documental & \multicolumn{2}{|c}{} \\
\hline Total General & 309 & 99,96 \\
\hline & & \\
\hline
\end{tabular}

Tabla I. Distribución de las referencias por asignatura. 
Las dos primeras asignaturas, Catalogación Automatizada y Catalogación, presentaron mayor número de referencias en sus planes de enseñanza, bien arriba de la media, que es de 13,43 referencias. Según consta, eso se da en función de ser asignaturas que presentan un contenido bastante pulverizado por los diversos campos, sin presentar una literatura más consistente.

Además, estudios realizados por el CAPES (Schleyer y Colonelli, 1978), sobre los planes de enseñanza de los 29 cursos de Biblioteconomía que había en Brasil, y que participaron en el proyecto que evaluó la situación de las escuelas de Biblioteconomía y Documentación indican que la "literatura recomendada en la enseñanza de Biblioteconomía, en nivel de licenciatura está excesivamente orientada a las asignaturas técnicas", tales como Catalogación (el 16,64\% en esos estudios del CAPES). Comparando los resultados de la investigación con éstos, se puede concluir que hubo poco o ningún cambio del cuadro.

Un conjunto bien grande de asignaturas, nueve en el total, demuestran un número de referencias menor del $2 \%$ ( 2 a 6 referencias), esto es, bien menor que la media de referencias, que es del $13,43 \%$.

Los formatos bibliográficos que predominaron en la literatura encontrada en los planes de enseñanza fueron los libros y capítulos de libros, que completaron el $51,46 \%$ del total, confirmando así la preferencia de este tipo de documento por las Ciencias Sociales, incluyendo ahí las Ciencias Sociales Aplicadas (Biblioteconomía).

Considerando que estas ciencias tienen un desarrollo más lento, en comparación con el área de Ciencias Exactas o Biológicas, por ejemplo, los libros constituyen una literatura con valor más duradero, contraponiéndose a las revistas, que constituyen una literatura más efímera. A pesar de esto, las revistas fueron referenciadas 95 veces, lo que representa el $30,74 \%$ del total, y puede ser considerada una presencia significativa.

Con relación a la lengua de las referencias (Tabla III), 202 de ellas fueron citadas en portugués, representando el $65,37 \%$ del total, predominando en las bibliografías, lo que es natural, tomando en consideración las limitaciones lingüísticas de los alumnos de licenciatura. La cantidad de material en lengua inglesa parece sorprendente -el $30,10 \%$ de las referencias, que es un porcentaje bastante representativo.

Al mismo tiempo, es también de extrañarse el bajo porcentaje de textos en español, considerando la aparentemente mayor facilidad que los alumnos tienen con esa lengua, y considerando que el español es una de las lenguas oficiales de varios organismos internacionales, tales como UNESCO y OEA, que editan varias publicaciones sobre Biblioteconomía, además de ser más accesible a los brasileños, por ser lengua latina.

\begin{tabular}{lcr}
\hline Tipo de material & $N^{\circ}$ de referencias & $\%$ \\
\hline Libros y capítulos de libros & 159 & 51,46 \\
Periódicos & 95 & 30,74 \\
Documentos electrónicos & 39 & 12,62 \\
Tesinas finales, Tesis y & 09 & 2,91 \\
Disertaciones & 04 & 1,29 \\
Literatura de eventos & 03 & 0,97 \\
Otros & & \\
\hline Total & 309 & 99,99 \\
\hline
\end{tabular}

Obs.: En la variable "otros" están englobadas referencias de ABNT; en la variable "documentos electrónicos", referencias de la Internet

Tabla II. Tipología de los documentos.

\begin{tabular}{lcr}
\hline Idioma & $N^{\circ}$ de referencias & $\%$ \\
\hline Portugués & 202 & 65,37 \\
Inglés & 93 & 30,10 \\
Español & 14 & 4,53 \\
\hline Total & 309 & 100 \\
\hline
\end{tabular}

Tabla III. Lengua de las referencias.

El análisis de las referencias reveló 95 (noventa y cinco) citas de revistas nacionales y extranjeras, en el formato impreso y electrónico, referentes a un total 28 títulos, con una media de 3,39 citas para cada periódico.

Se verificó la importancia de la revista Ciência da Informação para el área: de las 95 referencias, $20 \%$ fueron para la revista en cuestión, reafirmando así que, además de la variedad de asuntos que cubre, atiende a las asignaturas diversas del currículo. La segunda revista más citada fue Perspectivas em Ciência da Informação, de UFMG.

Obsérvese que 20 de los 28 títulos tuvieron citas abajo de la media, lo que confirma que un gran número de revistas $(71,42 \%)$ recibe un pequeño número de citas, como afirma la Ley de Bradford, según la cual hay un número pequeño de revistas con productividad máxima (gran número de citas) y un gran número de revistas, en las cuales la dispersión de artículos es grande (con bajo número de citas). 


\begin{tabular}{|c|c|c|}
\hline Nombre del Periódico & $\begin{array}{l}N^{\circ} d e \\
\text { ref. }\end{array}$ & $\%$ \\
\hline Ciência da Informação & 19 & 20 \\
\hline Perspectivas em Ciência da & 11 & 11,58 \\
\hline Informação & 10 & 10,53 \\
\hline Cataloging e Classification Quartely & 07 & 7,37 \\
\hline Revista Brasileira de Biblioteconomia e & 05 & 5,26 \\
\hline Documentação & 05 & 5,26 \\
\hline Transinformação & 05 & 5,26 \\
\hline Cadernos da FFC & 04 & 4,21 \\
\hline Library Collections, Acquisitions e & 03 & 3,16 \\
\hline Technical Services & 03 & 3,16 \\
\hline Revista de Biblioteconomia de Brasília & 03 & 3,16 \\
\hline Library Resources and Technical & 02 & 2,10 \\
\hline Services & 02 & 2,10 \\
\hline Library Journal & 02 & 2,10 \\
\hline Library Hi Tech & 01 & 1,05 \\
\hline Journal of the American Society for & 01 & 1,05 \\
\hline Information Science & 01 & 1,05 \\
\hline Journal of Education for Library and & 01 & 1,05 \\
\hline Information Science & 01 & 1,05 \\
\hline Library Trends: artigos selecionados & 01 & 1,05 \\
\hline Caderno de Biblioteconomia & 01 & 1,05 \\
\hline $\begin{array}{l}\text { Revista Digital de Biblioteconomia e } \\
\text { Ciência da Informacão }\end{array}$ & 01 & 1,05 \\
\hline SCIRE & 01 & 1,05 \\
\hline Informação e Sociedade: Estudos & 01 & 1,05 \\
\hline Investigación Bibliotecológica & 01 & 1,05 \\
\hline Art Libraries Journal & 01 & 1,05 \\
\hline Bol. Bibliog. Bibli. Munic & 01 & 1,05 \\
\hline Andrade & 01 & 1,05 \\
\hline Bulletin of Libraries & & \\
\hline Informação e Informação & & \\
\hline Elebtronic Library & & \\
\hline D-LIB Magazine & & \\
\hline Library Management & & \\
\hline $\begin{array}{l}\text { Datagramazero - Revista de Ciência } \\
\text { da Informação }\end{array}$ & & \\
\hline Information Technology and Libraries & & \\
\hline Total & 9 & 9,9 \\
\hline
\end{tabular}

Tabla IV. Periódicos más citados.

\begin{tabular}{lcc}
\hline Tipo de Formato & $N^{\circ}$ de referencias & $\%$ \\
\hline Impreso & 223 & 72 \\
Electrónico & 49 & 16 \\
Impreso y Electrónico & 37 & 12 \\
\hline Total & 309 & 100 \\
\hline
\end{tabular}

Tabla V. Formato de los documentos.

Destacan todavía, con un número de citas por encima de la media, las siguientes revistas: Cataloging e Classification Quartely (10 citas), Revista Brasileira de Biblioteconomia e Documentação (7), Transinformação (5), Cadernos da FFC (5), Library Collections, Acquisitions e Technical Services (5), y Revista de Biblioteconomia de Brasília (4), confirmándose así más una vez la Ley de Bradford. Obsérvese todavía el gran número de revistas en lengua extranjera, en inglés o español, siendo 19 de los 28 títulos $(67,85 \%)$.

\begin{tabular}{lcc}
\hline $\begin{array}{l}\text { Localidad de las } \\
\text { instituciones }\end{array}$ & $\begin{array}{c}N^{\circ} \text { de } \\
\text { referencias }\end{array}$ & $\%$ \\
\hline $\begin{array}{l}\text { Autores de instituciones } \\
\text { brasileñas }\end{array}$ & 217 & 62 \\
$\begin{array}{l}\text { Autores de instituciones } \\
\text { extranjeras }\end{array}$ & 135 & 38 \\
\hline Total & & \\
\hline
\end{tabular}

Tabla VI. Procedencia de los autores.

\begin{tabular}{lc}
\hline Autores & $\begin{array}{c}N^{\circ} \text { de } \\
\text { referencias }\end{array}$ \\
\hline Barbosa, Alice Príncipe & 05 \\
Fujita, Mariângela S.L. & 05 \\
Campello, Bernadete S. & 05 \\
Guimarães, José A.C. & 05 \\
Cintra, Ana M.M. & 05 \\
Yee, M.M. & 05 \\
Silveira, A. & 04 \\
Cunha, Murilo B.da & 04 \\
Rowley, Jennifer & 04 \\
Robredo, Jaime & 04 \\
Valentim, M.L.P. & 04 \\
Balby, Claudia N. & 04 \\
Amaral, S.A. do & 03 \\
Figueiredo, Nice M.de & 03 \\
Kremer, Jeannette M. & 03 \\
ABNT & 03 \\
Smit, Johanna W. & 03 \\
Hopkinson, Alan & 03 \\
Classificação Decimal de Dewey & 02 \\
Vergueiro, Waldomiro & 02 \\
Guinchat, C. & 02 \\
Menou, M. & 02 \\
Lancaster, F.W. & 02 \\
Pinto Molina & 02 \\
França, J.L. & 02 \\
Barreto, A.de A. & 02 \\
Mason, R.C.T. & 02 \\
Magalhães, M.H.A. & 02 \\
Dias, M.R.I. & 02 \\
Fiuza, M.M. & 02 \\
Foundations of Cataloguing & 02 \\
Hagler, R. & 02 \\
Hichey, D.J. & 02 \\
Jones, W. & 02 \\
\hline & \\
&
\end{tabular}

Tabla VII. Autores más citados.

En el formato impreso, la mayoría de las referencias está compuesta por libros y capítulos de libros y artículos de periódicos. En el formato 
electrónico, la mayoría de las referencias está compuesta por las revistas Revista Digital de Biblioteconomia e Ciência da Informação, D-LIB Magazine y Datagramazero, y por documentos de la Internet. En el formato impreso y electrónico, la mayoría de las referencias está compuesta por revistas que están disponibles en los dos formatos, entre ellas Transinformação, Ciência da Informação, Perspectivas em Ciência da Informação y Scire.

Obsérvese que las referencias con más de 2 autores recibieron cada una una cita en el proceso de tabulación, por eso el total es de 352, y no de 309, que es el total de referencias analizadas.

En una temporalidad de 0 a 57 años la vida media de la literatura es de 11 años. La literatura con edad de 3 años posee el mayor número de referencias (23), representando $7,44 \%$ del total de referencias.

De los autores más citados 5 de ellos proceden de la UNESP y cinco de ellos suceden de la USP

\section{Consideraciones Finales}

El análisis de los datos contribuye al conocimiento de la identidad del curso de Biblioteconomía de UNESP. A pesar de no constituir un estudio exhaustivo, tuvo como preocupación básica suministrar datos sobre la literatura utilizada para la formación de los bibliotecarios, futuros profesionales de la información, a través del análisis de algunos aspectos de los documentos referenciados en los planes de enseñanza.

Se sugiere que tal análisis sea rehecho con una cierta periodicidad, con la intención de disponer siempre de indicaciones sobre lo qué es citado, con el objetivo de obtenerse un perfil epistemológico del Curso.

\section{Referencias bibliográficas}

Brambilla, S. D. S.; Stumpf, I.R.C. (2006). Planos de ensino do Curso de Biblioteconomia da Universidade Federal do Rio Grande do Sul: estudo bibliométrico das referências. // Transinformação, Campinas. 18:1 (jan. labr., 2006) 37-47.

Dias, E. J. W.; Pitella, M. C.; Pontello, A. G. P. (1996). Literatura utilizada no ensino de graduação em Biblioteconomia no Brasil: produtividade institucional. // Perspectiva em Ci. Inf., Belo Horizonte, v. 1:2 (jul/dez., 1996) 157.

Lawani, S. M.; Baye, A. E. (1993). Validity of citacion criteria for assessing the influence of scientific publications: new evidence with peer assessment. // Journal of the AmericanSociety for Information Science. 34:1 (jan., 1993) 5966.

Silva, C. S.; Machado, L. M. Machado (1998). Nova LDB: Trajetória para a cidadania? São Paulo: Arte \& Ciência, 1998.

Schleyer, J. R.; Colonelli, C. A. (Coordenação de aperfeicoamento de pessoal de nível superior). $\mathrm{O}$ ensino de $\mathrm{Bi}$ blioteconomia no Brasil. Brasília: Capes, 1978, v.3: Análise da literatura recomendada no ensino de Biblioteconomia no Brasil. 